\title{
12 Das Sein als Schein (II): Die Verstetigung des Seins als Geltungsanspruch
}

Die Argumentationsschritte, mit welchen Hegel im zweiten Teil des „Schein“Abschnitts die Integration des Scheins in das Wesen leistet, interessieren uns hier nicht in aller Kleinteiligkeit, da sie zu unserer Fragestellung nichts Neues beitragen. Wichtig und zu erörtern ist einzig die prinzipielle Art und Weise, der Grund sowie das Ergebnis der scheinbar vollständigen Aufhebung des Scheins im Wesen, d.h. die Rahmenbedingungen und Konsequenzen dieses Vorgangs. Es ist nämlich von entscheidender Bedeutung, dass Hegel jetzt gerade nicht in (für die Logik) üblicher Weise vorgeht: also den Gegensatz von Schein und Wesen gemäß der sich aus seiner Gegensätzlichkeit ergebenden begrifflichen Widersprüche und logischen Inkonsistenzen im bekannten dreifachen Sinn aufzuheben. Denn die bisher bereits herausgearbeitete Pointe in Bezug auf den Schein ist es ja gerade, dass sein Bestehen trotz der vollständigen Aufhebung des Seins eben als Rückkehr gedacht werden muss: Hegel bestätigt dies indirekt, indem er nun die weitere Vorgehensweise ändert und dieser Einsicht anpasst. Denn der Schein als das ,an sich nichtige“ (GW 11, S. 247) kann nicht (erneut) aufgehoben werden, da er bereits vollständig aufgehoben ist, obwohl er ,eine unmittelbare Voraussetzung, eine unabhängige Seite gegen das Wesen“ (GW 11, S. 247) noch immer enthält; ein weiterer Aufhebungsversuch würde an diesem Resultat nichts ändern, da sich die Bedingungen bisher noch nicht geändert haben. Folglich gilt es, die übliche logische Bewegungsrichtung und ihren methodischen Kern beinahe umzukehren:

\footnotetext{
Es ist aber von ihm [dem Schein, C.W.], insofern er vom Wesen unterschieden ist, nicht zu zeigen, daß er sich aufhebt und in dasselbe zurückgeht; denn das Seyn ist in seiner Totalität in das Wesen zurückgegangen; der Schein ist das an sich nichtige; es ist nur zu zeigen, daß die Bestimmungen, die ihm vom Wesen unterscheiden, Bestimmungen des Wesens selbst sind, und ferner, daß diese Bestimmtheit des Wesens, welche der Schein ist, im Wesen selbst aufgehoben ist. (GW 11, S. 247)
}

Die Anerkennung der logischen Ansprüche, die das Residuum seinshafter Äußerlichkeit im Schein gerade in seiner vollständigen Negation geltend macht, wird auch darin ersichtlich, dass Hegel hier ein Vorgehen skizziert, welches den Schein nicht aufhebt, sondern als Schein bestehen lässt. Der Schein soll nicht umstandslos im Wesen verschwinden, sondern umgekehrt sollen die Charaktere des Scheins so neu beschrieben werden, dass ersichtlich ist, inwiefern diese nichts anderes als die Charaktere des Wesens sind. Hegel setzt also die Einsicht in die komplexe Bestehensweise des Scheins ganz konsequent auch in der Art und Weise um, wie dessen progressive Identifikation mit dem Wesen operativ aufzu- 
zeigen ist: indem nämlich das perennierende Bestehen des Scheins belassen und von diesem aus dessen Identität mit den Grundbestimmtheiten des Wesens festgestellt wird. Der Schein verschwindet nicht im Wesen: Das Wesen erweist sich als Inbegriff des Scheins, sodass der Schein nichts anderes als das Wesen ist, aber in der Form einer unaufhebbaren, von ihm gemeinten Äußerlichkeit zum Wesen. Auf genaueste Weise kehrt so die Figur des Scheins, im Raum seiner teleologischen Entwicklung zum Wesen hin, zugleich stets auf die Artikulation der Bedingungen des Wesens zurück, die es mit seinem Auftreten verkörpert. Genau an dieser Stelle erwächst aus der Struktur des Scheins die Möglichkeit seiner realitätskategorialen Funktion, die später ausführlicher beschrieben wird: nämlich als Einforderung der logischen Bedachtsamkeit gegenüber einem Bezugspunkt des „Bestimmens überhaupt“, der nicht anders denn als dem Bestimmen äußerlich zu denken ist, ohne dass er jenseits von Bestimmbarkeit liegt.

Es ist also die Strategie Hegels, die Bestimmungen des Scheins, welche ihn zu einer dem Wesen äußerlichen Bestimmung machen, als eigene Bestimmungen des Wesens aufzuzeigen, ohne zugleich die Differenz zwischen beiden auszulöschen $^{91}$. Dafür müssen die tragenden Charaktere des Scheins, (a) seine „Nichtigkeit“ und (b) seine „Unmittelbarkeit“, mit den tragenden Charakteren des Wesens, (a) seiner „Negativität“ und (b) seiner „Gleichheit mit sich selbst“ (GW 11, S. 247), identifiziert werden, um zu zeigen, dass die Charaktere des Scheins, in ihrer Wahrheit verstanden, nichts anderes als die inneren Bestimmungen des Wesens selbst sind und so der Schein gegen das Wesen eine Beziehung ist, die eigentlich als Selbstbeziehung des Wesens in sich selbst zu verstehen ist: „Der Schein ist also das Wesen selbst, aber das Wesen in einer Bestimmtheit, aber so daß sie nur sein Moment ist, und das Wesen ist das Scheinen seiner in sich selbst.“ (GW 11, S. 249) Die sehr gedrängten Wege dieser Identifikationsbewegung ${ }^{92}$ und die Probleme, die sich möglicherweise durch die semantischen Verschiebungen ergeben, die Hegel den Konzepten von Nichtigkeit und v.a. von Unmittelbarkeit im Übergang in Bestimmungen des Wesens aufzwingt ${ }^{93}$, brauchen uns hier, wie gesagt, nicht weiter zu interessieren. Für unsere Fragestellung bleibt festzuhalten: Das Verfahren nicht-aufhebender Integration des Scheins in das Wesen, wie es von Hegel hier entworfen worden ist, garantiert, dass auch im Wesen bzw. der Verinnerlichung des Scheins in das Wesen der bestimmte Geltungsanspruch des Scheins gegen das Wesen in den Bedingungshaushalt des Wesens Eingang findet. Seine Reformulierung in der Grammatik des Wesens be-

91 Vgl. Iber: Metaphysik absoluter Relationalität, S. $83 \mathrm{f}$.

92 Genauestens zeichnet Iber (Metaphysik absoluter Relationalität, S. 83-119) diese nach.

93 Vgl. dazu Henrich: Hegels Logik der Reflexion. 
hält die intensionale Beziehung auf eine Andersheit, deren Integration als nichtintegrierte die Bedingung für eben jenen kategorialen minimalen Realitätsbegriff abgibt, wie er dann in den Reflexionsformen ausformuliert werden wird.

An dieser Stelle kommt nun ein weiterer entscheidender Aspekt des Wesens hinzu, durch den erst das Verhältnis von Schein und Wesen weiter geklärt werden kann: nämlich der der selbstbezüglichen Negativität als Kernstruktur des Wesens, die es im Folgenden näher zu erläutern gilt und die insb. in dem Kapitel zu den Reflexionsformen (Kap. 13-15 im Hauptteil II) Berücksichtigung findet.

Diese logische Artikulation einer Bestimmungsform, die weder bloß gegen das Wesen besteht, noch bloß innerhalb des Wesens ihren logischen Platz findet, ist deshalb möglich, weil die Grammatik des Wesens in ihrer vollen Struktur eine begriffliche Stelle dafür vorgesehen hat, die das „Gegen“-das-Wesen-Bestimmtsein innerhalb des Wesens bestimmt und vertritt: und zwar deshalb, weil Hegel den begrifflichen Kern des Wesens überhaupt von der Idee selbstbezüglicher Negativität als „Abstoßen seiner von sich selbst“ zu entwickeln sucht. Erst die Reflexionsformen jedoch können hier als wirkliche Umsetzung dieser Strukturalität des Wesens unter Beachtung der Geltungsansprüche des Scheins betrachtet werden: Denn in ihnen aktualisieren sich die Strukturvorgaben selbstbezüglicher Negativität des Wesens (absolute Negativität und reflektierte Unmittelbarkeit in ihrer Identität als Wesen) unter Integration der Ansprüche des Scheins zu einer komplexen kategorialen Grundform von „Bestimmtsein überhaupt“, die zuallererst als minimale kategoriale Idee von Realität überhaupt gelten darf. Der Geltungsanspruch scheinhafter Äußerlichkeit gewinnt demnach erst in den Reflexionsformen eine logische Gestalt, indem er vollständig in wesenshafte Bestimmungen des Bestimmens und damit in operative kategoriale Begriffe des Wesens übersetzt wird. Im Folgenden sollen die Voraussetzungen dieser integrativen Transformation in den Reflexionsformen, nämlich die Resultate des „Schein“-Kapitels, die vor allem in (a) Hegels Entwicklung der Stufen von Negativität und (b) der Arten von Unmittelbarkeit liegen, etwas genauer beleuchtet werden, um so den argumentativen Schritt hin zur vollen Entfaltung der operativen Infrastruktur des Wesens in den Reflexionsformen im Sinne unserer Fragestellung darstellen zu können.

Herzstück dieses Resultats der Integration des Scheins in das Wesen im zweiten Teil des „Schein“-Kapitels sind die drei Formen von Negativität (und die ihr entsprechenden drei Formen der Unmittelbarkeit ${ }^{94}$ ), die in ihrem Zusam-

94 Von diesen her entwickelt Henrich (Hegels Logik der Reflexion, S. 114f.) diese Stufen, wohingegen Iber komplementär eher den Blick auf die zugeordneten Begriffe von Negativität richtet (Iber: Metaphysik absoluter Relationalität, S. 103-105, 112). 
menhang wiederum den Strukturzusammenhang des Wesens selbst bilden: und zwar dergestalt, dass das Wesen nach dem Aufweis der Integration des Scheins als Einheit von Unmittelbarkeit und Negativität sich denken lassen muss, d.h. seine Negativität nichts anderes sein darf als die Unmittelbarkeit des Seins, welche in transformierter wesenslogischer Form über die Stufe ihres reflektierten Bestehens als Schein nun als vollständige interne Bestimmung des Wesens reformuliert worden ist. ${ }^{95}$ Das Wesen als Nachfolger des Seins hat seine Unmittelbarkeit gerade darin, absolute Negativität zu sein; d.h. es gibt scheinbar keine seinslogische Unmittelbarkeit mehr, die etwas anderes als die Form reiner Vermittlung in der Negativität ist.

Denn das Wesen ist das Selbständige, das ist als durch seine Negation, welche es selbst ist, sich mit sich vermittelnd; es ist also die identische Einheit der absoluten Negativität und der Unmittelbarkeit. - Die Negativität ist die Negativität an sich; sie ist ihre Beziehung auf sich, so ist sie an sich Unmittelbarkeit; aber sie ist negative Beziehung auf sich, abstossendes Negiren ihrer selbst, so ist die an sich seyende Unmittelbarkeit das Negative oder Be stimmte gegen sie. Aber diese Bestimmtheit ist selbst die absolute Negativität und diß Bestimmen, das unmittelbar als Bestimmen das Aufheben seiner selbst, Rückkehr in sich ist. (GW 11, S. 248)

Erneut verdichtet sich die Argumentation zusehends. Es ist Christian Iber zu danken, dass er die schwierigen begrifflichen Bewegungen an dieser Stelle in seinem Kommentar genauestens nachgezeichnet und erschlossen hat. ${ }^{96}$ Grundgedanke ist es dabei, dass die Integration des Scheins in das Wesen durch die Identifikation ihrer jeweils tragenden Bestimmungen die Einheit der seinslogischen und der wesenslogischen Unmittelbarkeit in einer absoluten Negativität, die zugleich absolute Unmittelbarkeit ist, aus sich erzeugt. Hegel entwickelt an dieser Stelle bei genauerer Betrachtung drei Arten bzw. Stufen von Negativität und Unmittelbarkeit, die es auseinanderzuhalten und in ihrer Verbindung zu erörtern gilt.

1) Die sich auf sich beziehende Negativität des Wesens (,Negativität an sich“) ist, ganz für sich betrachtet, selbst eine Art von Unmittelbarkeit, weil sie in diesem reinen negativen Selbstbezug ganz in sich bleibt und so als „Gleichheit mit sich“ bzw. als Freiheit von der Beziehung auf Anderes ebenfalls „unmittelbar“ heißen kann - obwohl sie, anders als die seinslogische Unmittelbarkeit, weder diffe-

95 Henrich hat sich mit dieser Reformulierung seinslogischer Unmittelbarkeit kritisch auseinandergesetzt (Henrich: Hegels Logik der Reflexion, S. $111 \mathrm{ff}$.$) .$

96 Iber: Metaphysik absoluter Relationalität, S. 103-112. 
renzlos in sich noch „gegen die Vermittlung gefasst“97 ist. Damit ist sozusagen (allerdings natürlich im Rahmen der durchgängig nicht-formalen Logik Hegels gesprochen) die eher formallogische Seite der selbstbezüglichen Negativität in ihrem Charakter der Unmittelbarkeit beschrieben, indem hier rein auf die Form der Beziehung, nämlich als Beziehung der Sichselbstgleichheit, geachtet wird: Denn was sich nur auf sich bezieht (unabhängig von seinem substanziellen Gehalt), bezieht sich auf nichts anderes und bleibt damit in seinem Beziehen sich gleich, weshalb es „unmittelbar“ heißen darf.

2) Nimmt man dagegen den Gehalt dieser besonderen Art der Selbstbeziehung in den Blick, ergibt sich die zweite Form der Unmittelbarkeit des Wesens, die zur ersten in einem bestimmten Gegensatz steht, so wie sie selbst nur als Gegensatz zum Wesen zu denken ist. Denn das sich selbst negierende Negative ist „negative Beziehung auf sich, abstossendes Negieren ihrer selbst, so ist die an sich seyende Unmittelbarkeit das Negative oder Bestimm te gegen sie“ (GW 11, S. 248). Das sich selbst negierende Negative - hier greift Hegel auf die einfache formallogische Idee der doppelten Negation zurück - bedeutet im Resultat seines Vollzuges an sich selbst das Verschwinden des Negativen und das Setzen eines reinen Positiven, das als Anderes zur sich negierenden Negation - und damit ,gegen“ diese bestimmt ist. Das Negative, das sich negiert, setzt damit resultativ das reine Positive als Anderes gegen sich selbst: Das (seinslogisch) Andere aber zur reinen Negativität, d.h. zur Grundstruktur von Vermittlung und „Bestimmtheit überhaupt“, ist die reine Unmittelbarkeit. Im Unterschied zur seinslogischen Unmittelbarkeit zeigt sich nun hier, dass diese Unmittelbarkeit zugleich das Produkt einer Vermittlung - und damit dieser nicht bloß entgegengesetzt - ist. Sie ist deshalb reflektierte Unmittelbarkeit, die an sich den Gegensatz zur Vermittlung aufgehoben hat, weil sie selbst nur als Vermittlungsprodukt besteht, auch wenn sie das Vermittlungslose bedeutet. Diese Unmittelbarkeit bleibt zwar als gegen das Wesen bestimmte nun bestehen: Ihr Verständnis jedoch, d. h. die Entfaltung ihrer logischen Architektur wie Funktion, ist noch nicht vollendet.

3) Als Einheit der formalen Unmittelbarkeit des Wesens (1.) und der gehaltlichen Unmittelbarkeit gegen das Wesen (2.) ergibt sich jetzt auf der dritten Stufe ein adäquater wesenslogischer Begriff von Unmittelbarkeit, der dem Schein entspricht. Um als „absolute Negativität“ (GW 11, S. 248) verständlich zu sein, gilt es, den Zusammenhang der ersten und zweiten Ebene der Unmittelbarkeit im Wesen als dritte Ebene zu explizieren: „Aber diese Bestimmtheit ist selbst die absolute

97 Henrich: Hegels Logik der Reflexion, S. 111. Genau hier liegt die Bedeutungsverschiebung im Begriff der Unmittelbarkeit, aus der heraus Henrich fragt, ob diese Umbesetzung des Begriffs gerechtfertigt ist, ohne seine Grenzen gänzlich aufzulösen. 
Negativität und dies Bestimmen, das unmittelbar als Bestimmen das Aufheben seiner selbst, Rückkehr in sich ist.“ (GW 11, S. 248) Das Verhältnis der sich aus der selbstbezüglichen Negation ergebenden resultativen Unmittelbarkeit, die gegen die Negativität bestimmt ist, $z u$ dieser sich negierenden Negation, d. h. zu ihrem Ursprung, ist nicht bloß das eines Gegensatzes - schon gar nicht das eines ausschließenden. Das volle Begreifen ihrer Relation aber setzt bereits voraus, die Form des „Widerspruchs“, welche sich als volle Gestalt des Unterschieds erst später als Reflexionsbestimmung aus den Reflexionsformen ergeben wird (vgl. GW 11, S. 279-290), hier bereits zur Verfügung zu haben: Widerspruch beschreibt die Einheit des „Scheinen[s] seiner im andern“ und das „Setzen seiner als des andern“ (GW 11, S. 283) dergestalt, dass die sich Gegensätzlichen zugleich „das Ganze und sein Moment“ (GW 11, S. 266) der Gegensatzrelation einnehmen: „Die Entgegengesetzten enthalten insofern den Widerspruch, als sie in derselben Rücksicht sich negativ aufeinander beziehende oder sich gegenseitig aufhebende und gegeneinander gleichgültige sind.“(GW 11, S. 288) ${ }^{98}$ In eben diesem Sinne bilden selbstbezügliche Negation und resultative Unmittelbarkeit einen Widerspruch: Sie sind nicht nur als Andere zueinander bestimmt, sondern auch als Einheit und damit Gleichheit eines sich in diesem Gegensatz auf sich beziehenden und darin bei sich bleibenden Wesens. Denn da die Bestimmtheit der resultativen Unmittelbarkeit gegen die selbstbezügliche Negativität eben nur das Resultat dieser Negativität ist, d.h. sich als Vermittlungsprodukt aus ihrer doppelten Negation ergeben hat, kann sie eigentlich rein an sich selbst nichts anderes als diese Negativität sein. Damit aber ist sie „reflektierte Unmittelbarkeit“ und somit eben der Schein als in das Wesen integrierter, der jetzt der eigene Schein des Wesens ist, d. h. seine innere Beziehung auf sich als Anderssein, wo er zuvor noch gegen das Wesen zu bestehen schien.

Zugleich ist auch das Verhältnis der selbstbezüglichen Negativität zu der sich aus ihr ergebenden resultativen Unmittelbarkeit ein negatives - damit aber verwirklicht es eben jene Relation, die die selbstbezügliche Negativität schon an sich hat. Die operative Logik des Verhältnisses von resultativer Unmittelbarkeit (Produkt) und selbstbezüglicher Negativität (Produzierendes) fügt an allen drei Funktionsstellen die Identität der selbstbezüglichen Negativität ein: Die resultative Unmittelbarkeit ist an sich nichts anderes als die selbstbezügliche Negativität, weil sie logisch gesehen ihr Ergebnis ist bzw. das, was im ständigen Wechsel an ihre Stelle tritt; das Gegensatzverhältnis zwischen resultativer Unmittelbarkeit und selbstbezüglicher Negativität ist relational nichts anderes als die selbstbe-

98 Vgl. dazu genauer Claudia Wirsing: „Dialectics“, in: The Oxford Handbook of German Philosophy in the Nineteenth Century, hg.v. Michael N. Forster, Kristin Gjesdal, Oxford 2015, S. 651-673. 
zügliche Negativität, weil es ein negatives Verhältnis ist, in welchem sich die Negativität auf sich selbst (in Form der resultativen Unmittelbarkeit) bezieht (und damit ein Widerspruchsverhältnis bildet, das Inbegriff von Negativität ist). Damit jedoch kehrt die erste Unmittelbarkeit des Wesens, die Sich-selbst-Gleichheit des Negativen im Bezug auf sich selbst, ebenfalls zurück: Der Bezug der selbstbezüglichen Negation auf das sich aus ihr gegen sie ergebende Unmittelbare ist nichts anderes als das Sich-auf-sich-Beziehen der Negativität, in welchem die selbstbezügliche Negativität ganz bei sich bleibt im negativen Bezug auf ihr Anderes (die Unmittelbarkeit). Die Struktur absoluter Negativität ist erreicht: nicht nur darin, dass die Negativität in ihrem Anderen, der Unmittelbarkeit, ganz bei sich selbst und so im Abstoßen von sich zu sich zurückkehrt. Absolut ist sie auch dahingehend, dass Hegel den Kernprozess des Wesens, die selbstbezügliche Negativität, als ein Moment des diskutierten Verhältnisses in ihrer Funktionsweise auf jedes andere Moment des Verhältnisses ausweitet, sodass schließlich das Wesen nichts anderes ist als ein selbstbezügliches negatives Verhältnis, das sich in einem selbstbezüglichen negativen Verhältnis auf sich als anderes selbstbezügliches negatives Verhältnis (Unmittelbarkeit) bezieht: Radikaler und konzentrierter ist der Gedanke positionsloser negativer Vermittlung - man könnte sagen: des reinen Bestimmens und „Bestimmtseins überhaupt“ in seiner absolut-minimalen Grundverfassung - niemals gedacht worden. „Positionslos“ ist die reine selbstbezügliche Negativität, weil sie keinerlei externen Gegenstand hat, auf den sie sich bezieht, sondern die reine Grundform von „Vermittlung überhaupt“, isoliert von allen gegenständlichen Kontexten, darstellt.

Es ist deutlich geworden, dass die Integration des Scheins in das Wesen weder den Unterschied zwischen beiden verwischen, noch diesen Unterschied in einen neuen bloßen Gegensatz zu ihrer Einheit im Wesen hineindrängen darf. Die logische Grammatik des Wesens hebt zwar alles Unvermittelte, bloß gegen die Vermittlung Stehende in sich auf, sie übersetzt es aber dabei in Vermittlungs- und Bestimmungsansprüche, die auch ein Bestimmtes als gegen das absolute wesenslogische Bestimmen bestimmen können. Das Unmittelbare ist nicht unbestimmt als Anderes bzw. Äußeres zum absoluten wesenslogischen Bestimmen; zugleich aber ist es in der Rolle des Anderen zum wesenslogischen Bestimmen bestimmt. In der vollständigen Integration des Scheins in das Wesen durch Reinterpretation seiner Bestimmungen erlaubt die Vollständigkeit seiner Transposition gerade nicht, die mit dem Schein gemeinten Ansprüche an ein kategoriales Denken von Äußerlichkeit als legitimationslos auszuzeichnen. Die iterativ-regenerative Dynamik des Scheins macht es vielmehr notwendig, in der Integration des Scheins in das Beschreibungssystem des Wesens dessen Geltungsansprüche als sie selbst mit zu übernehmen. „Scheinen in ihm selbst“ heißt demnach für die Grammatik des Wesens: Die Ansprüche des Scheins sind so zu denken, dass sie 
deren Formulierbarkeit in Ausdrücken des Wesens zulassen, ohne thematisch bloß zur Funktion des Wesens herabgesetzt zu werden.

In dieser Hinsicht nämlich ist die Logik von „Bestimmtheit überhaupt“ im Wesen all-inklusiv, ohne gleichmachend zu sein: Vielmehr erlaubt sie es aufgrund ihrer besonderen minimalen Prozessbeschaffenheit selbstbezüglicher Negativität, die Kraft des Unterschieds wahrheitsgemäß so weit als möglich zu entfalten. Wie in der zweiten und dritten Stufe der Negativität sichtbar geworden ist, gilt die wesenslogische Form des selbstbezüglichen Unterscheidens als Kernzelle von wahrhaftem „Bestimmtsein überhaupt“, die im gegensatzgenerativen Unterscheiden zugleich in sich bleibt, nicht nur für das je Andere im Unterschied, sondern auch für das Andere zum Unterschied: D.h. die Bestimmung von „Bestimmtheit überhaupt“ im Wesen ist so zu denken, dass ihre Selbstbeziehung auf Gleiches im Unterscheiden identisch ist mit der Negation ihres bloßen Insichseins in diesem Vollzug. Daran zeigt sich für die noch zu entwickelnde Form minimaler kategorialer Bestimmtheit von „Realität überhaupt“ bereits eine entscheidende Einsicht: Das Verbleiben im Medium der eigenen Bestimmheit selbstbezüglicher Negativität ist undenkbar ohne seinen Bezugspunkt eines Anderen zum Bestimmtsein, der gleichwohl nicht außerhalb von „Bestimmtheit überhaupt“ gedacht werden kann. Weil Hegel die Wahrheit des Wesens aus dem logisch radikal reduzierten Nukleus selbstbezüglicher, rein relationaler Negativität ${ }^{99}$ als Minimalformation von „Vermittlung überhaupt“ und damit von „Bestimmtsein überhaupt“ entwickelt, ist es ihm möglich, auf komplexe Weise die kategoriale Minimalform des „Realen überhaupt“ als Zusammenhang von Bestimmungsrichtungen und Bestimmtheitsfeldern noch vor allen festen Entitätsvektoren (Subjekt, Objekt etc.) zu denken: dergestalt, dass Inklusion und Exklusion der Bestimmungsrichtungen auf vernünftige Weise differenziell und zugleich integrativ verbunden werden können. Die Entgegensetzung von Bestimmtheit und Unbestimmtheit, Negativität und Unmittelbarkeit, Begriff und Sein setzt die gemeinsame logische Materie einer begrifflichen „Bestimmtheit überhaupt“ voraus, um diesen Gegensatz vernünftig denken zu können, ohne dass damit die Dimension der Äußerlichkeit ihres Andersseins immer schon in der Einheit des absoluten begrifflichen Bestimmens verschwunden wäre: Der bestimmende Gegensatz des Unterschieds ist zugleich der bestimmte Gegensatz zum Unterschied als Gegensatz im Unterschied, ohne doch bloß durch den Unterschied zu sein. Das Andere zur selbstbezüglichen Negativität als ihr zugleich eigenes Setzungspro-

99 Vgl. Iber (Metaphysik absoluter Relationalität, S. $189 \mathrm{ff}$.), der überzeugend aufgezeigt hat, was es bedeutet, eine rein selbstbezügliche Relation ohne Relata als logische Grundstruktur zu denken. 
dukt wie unvermitteltes Anderssein ist nur der Schein eines Anderen. Nach dem, was wir bisher über den Schein festgestellt haben, heißt dies eben nicht nur, dass es als Anderes nur reflektiert, d. h. bloß in negierter Form als Anderes zum Wesen zu denken ist und eigentlich gänzlich und restlos in das Wesen fällt, nur Funktion seines Tuns ist. Es heißt eben auch - und die Logik der Reflexionsformen wird dies klar ausbuchstabieren -, dass in der wesenlosen Andersheit der Unmittelbarkeit gegen das Wesen, gerade dort, wo sie als ein Unterschied im Wesen aufgedeckt worden ist, in welchem sich das Wesen in sich auf sich selbst bezieht, der unüberwindbare seinshafte Geltungsanspruch von Äußerlichkeit, der vorher von Hegel mit dem Terminus „Schein“ dargestellt worden ist, wesenslogisch reformulierbar als er selbst erhalten bleiben muss. Das In-sich-Bleiben im begrifflichen „Bestimmen überhaupt“ ist nur so zu denken, dass es auf ein Anderes als das begriffliche Bestimmen bezogen ist, das freilich zugleich immer schon als von der Art begrifflichen Bestimmtseins gedacht werden muss, um ein Anderes zum Bestimmen und dessen setzenden Vermittlungen sein zu können. Diese Formation einer „unendliche[n] Bestimmtheit“ (GW 11, S. 248) ist so gerade nicht als Ausdruck einseitiger bloßer Immanenz zu denken, als welche das Wesen hier etabliert werden soll: Damit würde man die Umfassendheit ignorieren, die Hegel im Blick hat. Das

Aufzeigen, daß das Unwesentliche nur Schein [ist], und daß das Wesen vielmehr den Schein in sich selbst enthält, als die unendliche Bewegung in sich, welche seine Unmittelbarkeit, als die Negativität, und seine Negativität als die Unmittelbarkeit bestimmt und so das Scheinen seiner in sich selbst ist (GW 11, S. 249),

wird dann reduzierend bzw. einseitig missverstanden, wenn man nicht die komplexen Entwicklungsstufen des Anfangs der Wesenslogik im Blick hat, in denen Hegel gerade gegen die Gefahr einer bloßen Innerlichkeitskonzeption des Wesens und eines bloßen Konstruktivismus des Bestimmens vorgeht - und damit erst die Möglichkeit eröffnet, die Formation wesenslogischer Bestimmtheit als minimale Matrix von kategorialer „Realität überhaupt“ den sich erst später ergebenden entitätslogischen Unterschieden wie Subjekt/Objekt, Innerlichkeit/Äußerlichkeit etc. zugrunde zu legen. Dass die logische Subjektformation des Wesens nicht mit abstrakter Subjektivität und folglich mit dem alten, überwundenen Begriff einer Innerlichkeit des Wesens zusammenfallen darf, ist der dringlichste Imperativ zu Beginn der Wesenslogik; aus ihr (d.i. die Subjektformation des Wesens) aber ergibt sich die Möglichkeit, die von Hegel solchen Unterscheidungen streng vorgeordnete Logik des absoluten reflexiven Zusammenhangs von Bestimmtsein und Bestimmtwerden in einer bisher unmöglichen philosophischen Grundsätzlichkeit als konsequente und überzeugende Entfaltung eines minimalen kategorialen 
Realitätsbegriffs zu verstehen. Dabei erfüllt der Schein die wichtige Funktion, einen bestimmten Gehalt des Seins gegenüber seiner reibungslosen Aufhebung im Wesen auszusondern und ihm eine verzögerte regenerative Rhythmik der Integration zuzuweisen, durch welche dieser einen überdauernden Geltungsanspruch für sich gewinnt: sodass sich dieser Gehalt des Seins auch im zweiten Anlauf der Aufhebung des Seins (als Schein) in das Wesen dergestalt erhält, als er sich in wesenslogische Termini methodischer Reflexionsbedingungen übersetzt, die den ständigen Widerstand gegen die Verinnerlichung des „Bestimmtseins überhaupt“ im Wesen - und damit gegen die Bedingung der Subjektivierung des Bestimmens - garantieren. Gerade im Verschwinden bloßer seinshafter Äußerlichkeit als Operationsfeld von „Bestimmtheit überhaupt“ im Wesen bleibt die unauslöschliche logische Erinnerung an den Schein zurück und bringt sich so zur Geltung, dass sie in ihrem Verschwundensein Bestand hat: als Scheinen des Wesens in ihm selbst. Derart ist das Unabgegoltensein dieser Äußerlichkeit gerade Funktion ihres Verschwundenseins: Nur als Verschwundene kann sie in der Grammatik des Wesens zu einem unabgegoltenen Geltungsanspruch werden, dem sich die Infrastruktur des Wesens beständig zu stellen hat. Die Reflexionsformen als vollständige Ausformulierung der wesenslogischen Generativität von „Bestimmtheit überhaupt“ werden dies in vollem Umfang zur Geltung bringen und damit als die gesuchte kategoriale Minimalform von „Realität überhaupt“ fungieren. 